\title{
SELECTIONS AND ORDERABILITY
}

\author{
JAN VAN MILL AND EVERT WATTEL
}

\begin{abstract}
Let $X$ be a compact Hausdorff space. Then $X$ has a selection if and only if $X$ is orderable.
\end{abstract}

0. Introduction. Let $X$ be a compact Hausdorff space and let $2^{X}$ denote the hyperspace of nonempty closed subsets of $X$. A selection for $X$ is a continuous map $F: 2^{X} \rightarrow X$ such that $F(A) \in A$ for all $A \in 2^{X}$. Let $X(2)$ denote the 2 -fold symmetric product of $X$, i.e. the subspace of $2^{X}$ consisting of all nonempty closed subspaces of $X$ containing at most two points. A weak selection for $X$ is a continuous map $s: X(2) \rightarrow X$ such that $s(A) \in A$ for all $A \in X(2)$. It is easy to see that $X$ has a weak selection if and only if there is a continuous map $s: X^{2} \rightarrow X$ such that for all $x, y \in X$,

(1) $s(x, y)=s(y, x)$, and

(2) $s(x, y) \in\{x, y\}$.

Such a map $s: X^{2} \rightarrow X$ will also be called a weak selection.

Michael [M] showed that for a continuum $X$ the following statements are equivalent: (a) $X$ has a selection, (b) $X$ has a weak selection, and (c) $X$ is orderable. In [Y], Young claims, without giving a proof, that statements (a), (b), and (c) are also equivalent for compact zero-dimensional spaces $X$. In this paper we will show that, for compacta, statements (a), (b), and (c) are always equivalent.

1. The construction. Let $X$ be compact and let $s: X^{2} \rightarrow X$ be a weak selection. For each $x \in X$ define

$$
B_{x}=\{y \in X \mid s(y, x)=y\}
$$

and

$$
A_{x}=\{y \in X \mid s(y, x)=x\} \text {. }
$$

Observe that both $A_{x}$ and $B_{x}$ are closed, that $A_{x} \cup B_{x}=X$ and that $A_{x} \cap B_{x}=$ $\{x\}$.

1.1. TheOREM. Let $X$ be a compact space. Then the following statements are equivalent:

(a) $X$ is orderable,

(b) $X$ has a weak selection,

(c) $X$ has a selection.

Received by the editors October 3, 1980 and, in revised form, February 26, 1981.

1980 Mathematics Subject Classification. Primary 54F05; Secondary 54C65.

Key words and phrases. Compact ordered space, selection, weak selection. 
Proof. The implication (c) $\Rightarrow(b)$ is trivial and the implication (a) $\Rightarrow$ (c) is well known. Indeed, simply define $F: 2^{X} \rightarrow X$ by $F(A)=\min (A)$. An easy check shows that $F$ is a selection. It therefore suffices to prove that $(\mathrm{b}) \Rightarrow(\mathrm{a})$. To this end, let $s$ : $X^{2} \rightarrow X$ be a weak selection for $X$ and, for each $x \in X$, let $A_{x}$ and $B_{x}$ be defined as above. Let $\prec$ be a wellordering on $X$. For every $x \in X$ we will construct closed sets $L_{x}, U_{x} \subset X$ such that

(1) $L_{x} \cup U_{x}=X$ and $L_{x} \cap U_{x}=\{x\}$,

(2) if $y \prec x$ and if $x \in L_{y}$ then $L_{x} \subset L_{y} \backslash\{y\}$,

(3) if $y \prec x$ and if $x \in U_{y}$ then $U_{x} \subset U_{y} \backslash\{y\}$,

(4) if $z \in L_{x}$ and if $z \notin \cup\left\{L_{y} \mid y \prec x \& x \in U_{y}\right\}$ then $z \in B_{x}$,

(5) if $z \in U_{x}$ and if $z \notin \cup\left\{U_{y} \mid y \prec x \& x \in L_{y}\right\}$ then $z \in A_{x}$.

(In the total ordering on $X$ which we will construct in this proof, $L_{x}$ will be the set of all points smaller than or equal to $x$, and $U_{x}$ will be the set of all points larger than or equal to $x$.)

Let $x_{0}$ be the first element of $X$ and define $L_{x_{0}}=B_{x_{0}}$ and $U_{x_{0}}=A_{x_{0}}$. Assume that we have defined $L_{y}$ and $U_{y}$ for all $y \prec x$ satisfying (1) through (5). Let $E=\{y \prec$ $\left.x \mid x \notin L_{y}\right\}$ and $F=\left\{y \prec x \mid x \notin U_{y}\right\}$. Put

$$
Z=X \backslash\left(\bigcup_{y \in E} L_{y} \cup \bigcup_{y \in F} U_{y}\right) .
$$

Let $\kappa=|E|$ and for each $\xi \leqslant \kappa$ define points $y_{\xi} \in E$ in the following way:

(6) $y_{0}=\min (E)$,

(7) $y_{\zeta}=\min \left[\{x\} \cup\left\{y \in E \mid\left(y_{\mu} \prec y\right.\right.\right.$ for all $\left.\left.\left.\mu<\zeta\right) \&\left(y \notin \cup_{\mu<\zeta} L_{y_{\mu}}\right)\right\}\right]$. Let $\xi \leqslant \kappa$ be the first ordinal for which $y_{\xi}=x$.

Claim 1. If $\xi_{0} \leqslant \xi$ then $\cup\left\{L_{y} \mid y \in E \& y<y_{\xi_{0}}\right\}=\cup_{\mu<\xi_{0}} L_{y_{\mu}}$.

Take $y \in\left\{z \in E \mid z<y_{\xi_{0}}\right\} \backslash\left\{y_{\mu} \mid \mu<\xi_{0}\right\}$ and let $\mu \leqslant \xi_{0}$ be the first ordinal for which $y \prec y_{\mu}$. Since $y_{\rho} \prec y$ for all $\rho<\mu$ (notice that $\mu \neq 0$ ) and since $y \neq y_{\mu}$, by (7), $y \in \cup_{\rho<\mu} L_{y_{\rho}}$. Choose $\rho<\mu$ such that $y \in L_{y_{\rho}}$. Since $y_{\rho}<y$, by (2),

$$
L_{y} \subset L_{y_{\rho}} \subset \bigcup_{\delta<\xi_{0}} L_{y_{\delta}} .
$$

Claim 2. If $\mu_{0}<\mu_{1}<\xi$ then $L_{y_{\mu_{0}}} \subset L_{y_{\mu_{1}}} \backslash\left\{y_{\mu_{1}}\right\}$.

By (7), $y_{\mu_{1}} \notin L L_{\mu_{0}}$. Consequently, $y_{\mu_{1}} \in U_{y_{\mu_{0}}}$ and therefore, by (3), $U_{y_{\mu_{1}}} \subset$ $U_{y_{\mu_{0}}} \backslash\left\{y_{\mu_{0}}\right\}$. Consequently, by (1), $L_{y_{\mu_{0}}} \subset L_{y_{\mu_{1}}} \backslash\left\{y_{\mu_{1}}\right\}$.

Claim 3. If $\mu_{0}<\mu_{1}<\xi$ then $L_{y_{\mu_{1}}} \backslash L_{y_{\mu_{0}}} \subset A_{y_{\mu_{0}}}$.

Take $t \in L_{y_{\mu_{1}}} \backslash L_{y_{\mu_{0}}}$. Since $t \in U_{y_{\mu_{0}}}$ and, by (5),

$$
U_{y_{\mu_{0}}} \subset \bigcup\left\{U_{y} \mid y \prec y_{\mu_{0}} \& y_{\mu_{0}} \in L_{y}\right\} \cup A_{y_{\mu_{0}}},
$$

we may assume, without loss of generality that $t \in U_{z}$ for certain $z \prec y_{\mu_{0}}$ with $y_{\mu_{0}} \in L_{z}$; we will reach a contradiction. Assume that $y_{\mu_{1}} \in L_{z}$. Since $y_{\mu_{0}} \prec y_{\mu_{1}}$ and since $z \prec y_{\mu_{0}}$ this implies by (2), that $L_{y_{\mu_{1}}} \subset L_{z} \backslash\{z\}$. Consequently, $t \in L_{z} \backslash\{z\}$ and $t \in U_{z}$, contradicting (1). This shows that $y_{\mu_{1}} \notin L_{z}$ which implies that $y_{\mu_{1}} \in$ $U_{z}$. Since $z \prec y_{\mu_{1}}$, by (3), $U_{y_{\mu_{1}}} \subset U_{z}$ and therefore $x \in U_{z}$. If also $x \in L_{z}$ then $x=z$ which is impossible since $z \prec x$. We conclude that $x \notin L_{z}$ or equivalently, 
$z \in E$. Let $\varepsilon \leqslant \mu_{0}$ be the smallest ordinal such that $z \leqslant y_{e}$. Since $y_{\delta}<z$ for every $\delta<\varepsilon$ by (7), either $z=y_{\varepsilon}$ or $z \in l_{y_{\delta}}$ for certain $\delta<\varepsilon$. If $z=y_{\varepsilon}$ then $y_{\mu_{0}} \in L_{y_{\varepsilon}}$ which contradicts $z<y_{\mu_{0}}$ (Claim 2). Therefore, $z \in L_{y_{b}}$ for certain $\delta<\varepsilon$. Then $z \in L_{y_{8}} \subset$ $L_{y_{\mu_{0}}} \backslash\left\{y_{\mu_{0}}\right\}$. Since $z<y_{\mu_{0}}$ and since $y_{\mu_{0}} \in L_{z}$, by (2), we also have that

$$
L_{y_{\mu_{0}}} \subset L_{z} \backslash\{z\}
$$

which implies that $z \in L_{y_{\mu_{0}}} \subset L_{z} \backslash\{z\}$, a contradiction.

Claim 4. If $t \in \mathrm{Cl}_{X}\left(\cup_{y \in E} L_{y}\right) \backslash \cup_{y \in E} L_{y}$ then $t$ is a cluster point of the net $\left\{y_{\mu} \mid \mu<\xi\right\}$.

Suppose not and take a closed neighborhood $C$ of $t$ which misses

$$
\mathrm{Cl}_{\boldsymbol{x}}\left\{y_{\mu} \mid \mu<\xi\right\} \text {. }
$$

From Claim 1 it is clear that there is a cofinal subset $G \subset \xi$ with the property that for each $\mu \in G$ there exists a point $c_{\mu} \in C \cap L_{y_{\mu}}$ such that

$$
\mu=\min \left\{\delta<\xi \mid c_{\mu} \in L_{y_{8}}\right\} \text {. }
$$

Take $\mu \in G$. We claim that $c_{\mu} \in B_{y_{\mu}}$. If not, then by (4) there is a $y<y_{\mu}$ such that $c_{\mu} \in L_{y}$ and $y_{\mu} \in U_{y}$. Since $y \prec y_{\mu}$ and $y_{\mu} \in U_{y}$, by (3), $U_{y_{\mu}} \subset U_{y} \backslash\{y\}$ which implies that $L_{y} \subset L_{y_{\mu}}$. Consequently, $x \notin L_{y}$, since $x \notin L_{y_{\mu}}$, or equivalently, $y \in E$. By Claim 1 we can find $\delta<\mu$ such that $c_{\mu} \in L_{y_{b}}$, which is a contradiction since $\mu=\min \left\{\delta<\xi \mid c_{\mu} \in L_{y_{\delta}}\right\}$. This implies that for all $\mu \in G$ we have that $s\left(c_{\mu}, y_{\mu}\right)=$ $c_{\mu}$.

Let $(c, y)$ be a cluster point of the net $\left\{\left(c_{\mu}, y_{\mu}\right)\right\}_{\mu \in G}$. Then $c \in C$ and $y \notin C$, and since $s\left(c_{\mu}, y_{\mu}\right)=c_{\mu} \in C$ for all $\mu \in G$ it is clear that $s(c, y)=c$. Next take $\mu \in G$ arbitrarily. For all $\delta>\mu$ we have by Claim 3 that $s\left(y_{\mu}, c_{\delta}\right)=y_{\mu}$. Hence $s\left(c, y_{\mu}\right)=$ $s\left(y_{\mu}, c\right)=y_{\mu}$. This would imply that $s(c, y)=y$, and since $y \neq c$ this is a contradiction.

Claim 5. If both $t$ and $u$ are cluster points of the net $\left\{y_{\mu} \mid \mu<\xi\right\}$ then $t=u$.

Let $C$ and $D$ be closed and disjoint neighborhoods of, respectively, $t$ and $u$. There is clearly a cofinal subset $G \subset \xi$ and for each $\mu \in G$ points

$$
c_{\mu} \in C \cap\left\{y_{\lambda} \mid \lambda<\xi\right\} \text { and } d_{\mu} \in D \cap\left\{y_{\lambda} \mid \lambda<\xi\right\}
$$

such that if $\mu, \delta \in G$ and $\mu<\delta$ then

$$
c_{\mu}<d_{\mu} \prec c_{\delta} .
$$

Let $\left(t^{\prime}, u^{\prime}\right)$ be a cluster point of the net $\left\{\left(c_{\mu}, d_{\mu}\right)\right\}_{\mu \in G}$, then $t^{\prime} \in C$ and $u^{\prime} \in D$. By Claim $3, s\left(c_{\mu}, d_{\mu}\right)=c_{\mu}$ and consequently, $s\left(u^{\prime}, t^{\prime}\right)=t^{\prime}$. Fix $\mu \in G$. For each $\delta>\mu$ it is clear that $s\left(d_{\mu}, c_{\delta}\right)=d_{\mu}$ (Claim 3). Since $t^{\prime} \in \mathrm{Cl}_{X}\left\{c_{\delta} \mid \delta>\mu\right\}$ this implies that

$$
s\left(d_{\mu}, t^{\prime}\right)=d_{\mu} .
$$

Since $\left(u^{\prime}, t^{\prime}\right) \in \mathrm{Cl}_{X^{2}}\left\{\left(d_{\mu}, t^{\prime}\right) \mid \mu \in G\right\}$ this implies that $s\left(u^{\prime}, t^{\prime}\right)=u^{\prime}$. Since $u^{\prime} \neq t^{\prime}$, this is a contradiction.

Claim 6. $\cup_{y \in E} L_{y}$ has at most one boundary point.

Follows immediately from Claims 4 and 5.

Claim 7. If $t \in Z$ and $\mu<\xi$ then $t \in A_{y_{\mu}}$. 
Since $t \notin L_{y_{\mu}}$ clearly $t \in U_{y_{\mu}}$. T. erefore by (5), if $t \notin A_{y_{\mu}}$ then $t \in U_{y}$ for certain $y \prec y_{\mu}$ with $y_{\mu} \in L_{y}$. If $x \in L_{y}$ then $x \notin U_{y}$ since $x \neq y$ in which case $Z \cap U_{y}=$ $\varnothing$ which contradicts $t \in Z \cap U_{y}$. Therefore $y \in E$. By Claim 1

$$
\bigcup\left\{L_{y} \mid y \in E \& y \prec y_{\mu}\right\}=\bigcup_{\delta<\mu} L_{y_{\delta}} .
$$

Therefore $y_{\mu} \in L_{y_{\delta}}$ for certain $\delta<\mu$ which contradicts (7).

Formally we have to consider two cases, namely that $\xi$ is a successor or that $\xi$ is a limit ordinal. Those two cases can be treated analogously and since the case that $\xi$ is a limit is more complicated we will assume from now on that $\boldsymbol{\xi}$ is a limit.

Since $L_{y_{\mu}} \backslash\left\{y_{\mu}\right\}$ is open for each $\mu<\xi$, by Claims 1 and $2, \cup_{y \in E} L_{y}$ must have a limit point, say $a$, and by Claim 6 we see that $a$ is unique. By using precisely the same technique as above and again restricting our attention to the limit case we can find a limit ordinal $\eta$ and for each $\mu<\eta$ a point $z_{\mu} \in F$ such that

(8) if $\mu<\delta$ then $U_{z_{\mu}} \subset U_{z_{\delta}}$,

(9) $\cup_{\mu<\eta} U_{z_{\mu}}=\cup_{y \in F} U_{y}$, and

(10) if $t \in Z$ and $\mu<\eta$ then $t \in B_{z_{\mu}}$.

Again we find that $\cup_{y \in F} U_{y}$ has a unique boundary point, say $b$, and that this point is a cluster point of the net $\left\{z_{\mu} \mid \mu<\eta\right\}$.

(Note that, by (1), (2) and (3), $y \in E$ and $y^{\prime} \in F$ implies that $L_{y} \cap U_{y^{\prime}}=\varnothing$.)

Case 1. $a=b$. We then claim that $Z=\{x\}=\{a\}=\{b\}$. For assume that $t \in Z$. By Claim 7, $s\left(y_{\mu}, t\right)=y_{\mu}$ for all $\mu<\xi$ and consequently $s(a, t)=a$ since $a$ is a limit point of $\left\{y_{\mu}\right\}_{\mu<\xi}$. On the other hand, by $(10), s\left(t, z_{\mu}\right)=t$ for all $\mu<\eta$. By the same argument $s(t, a)=s(t, b)=t$. Hence $t=a$.

We therefore conclude that $a=b=x$ and that $Z=\{x\}$. Now define

$$
L_{x}=\bigcup_{y \in E} L_{y} \cup\{x\} \text { and } U_{x}=\bigcup_{y \in F} U_{y} \cup\{x\}
$$

An easy check shows that our inductive hypotheses are satisfied.

Case 2. $a \neq b$ and $x \notin\{a, b\}$. Define $L_{x}=\cup_{y \in E} L_{y} \cup\left(Z \cap B_{x}\right)$ and $U_{x}=$ $\cup_{y \in F} U_{y} \cup\left(Z \cap A_{x}\right)$. Observe that both $L_{x}$ and $U_{x}$ are closed since $a \in Z \cap B_{x}$ and $b \in Z \cap A_{x}$. Again an easy check shows that our inductive hypotheses are satisfied.

Case 3. $x=a$ and $a \neq b$. Define $L_{x}=\cup_{y \in E} L_{y} \cup\{x\}$ and $U_{x}=\cap_{\mu<\xi} U_{y_{\mu}}$.

Case 4. $x=b$ and $a \neq b$. Similar to Case 3.

Now define $x \leqslant y$ iff $x \in L$. Then $\leqslant$ is a linear order which generates the topology of $X$ since $X$ is compact and since for each $x \in X$ the sets $\{y \in X \mid y<$ $x\}$ and $\{y \in X \mid x \leqslant y\}$ are closed.

2. Notes. A space $X$ is called weakly orderable (abbreviated KOTS) provided that there is a linear order $\leqslant$ on $X$ such that for each $y \in X$ the sets $\{x \in X \mid x<$ $y\}$ and $\{x \in X \mid y \leqslant x\}$ are both closed. It is easily seen that whenever $X$ is a KOTS then the function $s: X^{2} \rightarrow X$ defined by $s(x, y)=\min \{x, y\}$ is a weak selection. This suggests the following question: 
Question. Let $X$ be a space. Is $X$ a KOTS if and only if $X$ admits a weak selection?

The technique used in the proof of our theorem is not applicable to answer this question since certain transfinite sequences of points need not have limit points.

\section{REFERENCES}

[M] E. Michael, Topologies on spaces of subsets, Trans. Amer. Math. Soc. 71 (1951), 152-182.

[Y] G. S. Young, Continuous selections on locally compact separable metric spaces, Lecture Notes in Math., vol. 171, Springer-Verlag, Berlin and New York, 1970, pp. 102-110.

Wiskundig Seminartum, Vrie Universiteit, De Borlelann 1081, 1081hV Amsterdam, The NETHERLANDS 